\title{
El diccionario bilingüe online en el proceso de enseñanza-aprendizaje del alemán como lengua extranjera. Una reflexión sobre hábitos y competencias en el sistema universitario español \\ María Egido Vicente (mariaegido@usal.es) \\ Universidad de Salamanca
}

\begin{abstract}
The development of electronic lexicography has led to the emergence of a new generation of lexicographic resources increasingly adapted to the specific needs of users, among which the online bilingual dictionary stands out. In this article, based on the specific case of German as a foreign language and from our own teaching experience, we reflect on the actual incidence of the online format in the lexicographic habits of users in the context of higher education. After the description of the fundamental properties of the digital resource and the presentation of concrete examples, the final aim is to open a discussion among foreign language teaching professionals, specifically for German as a foreign language, about the need to introduce a pedagogical lexicography in the university classrooms.
\end{abstract}

Keywords: electronic lexicography, bilingual dictionary, German as a foreign language, pedagogical lexicography, lexicographical competences.

\section{Resumen}

El desarrollo de la lexicografía electrónica ha propiciado la aparición de una nueva generación de recursos lexicográficos cada vez más adaptados a las necesidades concretas de los usuarios, entre los que destaca el diccionario bilingüe online. En este trabajo, partiendo del caso concreto del alemán como lengua extranjera y desde la propia experiencia docente, reflexionamos sobre la incidencia real del formato online en los hábitos lexicográficos de los usuarios en el contexto de la enseñanza superior. Tras la descripción de las propiedades fundamentales del recurso digital y la exposición de ejemplos concretos, el objetivo final es abrir una discusión entre los profesionales de la enseñanza de lenguas extranjeras y, en concreto, 
del alemán como lengua extranjera, en torno a la necesidad de incorporar una pedagogía lexicográfica en las aulas universitarias.

Palabras clave: lexicografía electrónica, diccionario bilingüe, alemán como lengua extranjera, pedagogía lexicográfica, competencias lexicográficas.

\section{INTRODUCGIÓN ${ }^{1}$}

Como en el resto de disciplinas científicas, la incorporación de las nuevas tecnologías al campo de la lingüística se desarrolla de forma paulatina. En el ámbito de la lexicografía, los inicios de lo que hoy se conoce como lexicografía electrónica o lexicografía digital se remontan a finales de los años 60 con la transliteración manual a cinta perforada del Webster's Seventh New Collegiate Dictionary (Gove 1963) a manos de Olney y Revard (Wilks et al. 1996: 81). Esta técnica derivó en la elaboración de los primeros diccionarios electrónicos con fines computacionales (machine-readable dictionaries), cuyo máximo exponente durante finales de los años 70 y la década de los 80 fue el Longman Dictionary of Contemporary English (Procter 1978), fuente principal de numerosas investigaciones en el campo del procesamiento del lenguaje natural (natural language processing, en lo sucesivo PLN). A partir de este momento, con la creación de bases de datos, se facilita el trabajo de los investigadores, quienes ya no tienen que hacer uso de las cintas perforadas para poblar los componentes léxicos de los sistemas de PLN (de Schryver 2003: 145). El desarrollo de los sistemas de almacenamiento y manipulación de datos, así como de nuevas bases de datos, propició la elaboración de los primeros diccionarios electrónicos para el consumo humano (human-readable electronic dictionaries), disponibles para el gran público desde finales de los años 80 .

En la actualidad y en consonancia con los avances llevados a cabo en el ámbito de la lexicografía digital durante la última década, el concepto de diccionario electrónico adquiere un significado amplio, ya que, tal y como afirma Nesi (2008: 458), "[it] can be used to refer to any data collection in electronic form concerned with the spelling, meaning or use of words," incluyendo sistemas automáticos de procesamiento de datos, glosarios, traductores o correctores ortográficos incorporados en programas de software. 
En el presente trabajo limitamos el concepto de diccionario electrónico a lo que comúnmente se conoce como diccionario online, accesible mediante dispositivos electrónicos como ordenadores, tabletas o móviles a través de internet. Dentro de este grupo nos centramos en los diccionarios bilingües online (DBO) dirigidos a aprendices hispanohablantes de alemán como lengua extranjera (DaF).

En esta relación, afirmar que el diccionario bilingüe (DB) es una herramienta de uso frecuente en el proceso de enseñanzaaprendizaje de DaF no supone, a día de hoy, ninguna revelación (cf. Albrecht 2005 Engelberg y Lemnitzer 2009, Albrecht 2005, Nied Curcio 2015). Dentro de los DB, el formato online o electrónico se ha convertido en el preferido de los aprendices, algo que, como se fundamenta empíricamente en varios trabajos desarrollados en el marco de la Wörterbuchbenutzungsforschung, ${ }^{2}$ se hace también extensible al contexto universitario español (Domínguez Vázquez et al. 2013: 146, Meliss 2015: 404).

Partiendo de esta premisa y de nuestra propia experiencia docente, nos planteamos lo siguiente: ¿qué ha supuesto la incorporación de las nuevas tecnologías al ámbito de la lexicografía?, ¿de qué manera ha influido en los usuarios, aquí aprendices hispanohablantes de DaF? y, lo más importante, ¿ha tenido alguna repercusión en sus hábitos de consulta? Este artículo tiene como objetivo profundizar en estas cuestiones con el fin de abrir una discusión en torno al uso de los DBO en el caso específico de los aprendices hispanohablantes de $\mathrm{DaF}$ en el marco de la enseñanza superior, sobre todo tras la llegada a las aulas de las primeras generaciones de nativos digitales, a los que, en principio, se les presupone un domino absoluto del medio virtual.

\section{LA ENSEÑANZA DE DAF EN EL SISTEMA EDUCATIVO UNIVERSITARIO ESPAÑOL: UNA APROXIMAGIÓN}

Desde la puesta en marcha del EEES, el sistema educativo universitario español, como en el resto de estados miembros de la UE, ha sufrido una restructuración paulatina acorde a los principios de 
calidad, diversidad, movilidad y competitividad formulados en la Declaración de Bolonia (1999). Este cambio de paradigma incide también en la percepción general que los diferentes agentes de la comunidad universitaria tienen del desarrollo de la competencia comunicativa en una o varias lenguas extranjeras como una de las competencias clave para la realización y desarrollo personal, así como para la inclusión social y laboral en el marco de una sociedad globalizada. ${ }^{3}$

En el caso concreto de la lengua alemana, y tal y como indica el informe "El español en Alemania. El alemán en España" (Martín Torres 2010: 251), "la transición paulatina de las carreras en el marco del Proceso de Bolonia ha tenido efectos sobre la oferta docente de alemán." En la actualidad, aparte de las carreras propias de la especialidad como Filología Alemana o Traducción e Interpretación, el alemán como segunda o tercera lengua extranjera se incluye en los planes de estudio de diversas titulaciones como Turismo, Empresariales, Filosofía, Humanidades, etc., a lo que se suma la oferta lingüística de los centros de lenguas de las universidades abiertos a oyentes de todas las facultades (Martín Torres 2010: 251). Los diferentes accesos a la enseñanza del alemán en el sistema universitario, ya sea por el carácter obligatorio u optativo de las asignaturas, el número de horas lectivas contemplado para cada titulación o la exigencia de unos conocimientos lingüísticos mínimos, dificulta la delimitación de un perfil específico del estudiantado universitario de DaF. Por ello, la reflexión que se persigue con la realización de este trabajo se fundamenta, además de en investigaciones específicas en el marco de la Wörterbuchbenutzungsforschung, en la observación de una muestra intencional de estudiantes del Grado en Turismo de la Universidad de Salamanca que eligen el alemán como segunda lengua extranjera obligatoria.

\section{APRENDICES DAF Y DBO, ¿POR QUÉ LOS UTILIZAN?}

La predilección por el formato online por parte de los estudiantes universitarios a la hora de seleccionar un diccionario se sucede en consonancia con la incorporación de los recursos digitales al proceso de enseñanza de la lengua extranjera, en este caso DaF (Höfler 2017: 
147 ss.), y, en definitiva, a la omnipresencia de lo virtual en su vida diaria. Acciones más o menos cotidianas hasta la llegada del nuevo milenio como comprar un billete de avión o reservar un viaje en una agencia física o, por ejemplo, esperar hasta el final del telediario para ver el parte meteorológico son, hoy en día, al menos para un amplio sector de la sociedad, acciones propias de otro tiempo. Lo mismo sucede en el ámbito académico con tareas cada vez menos frecuentes como fotocopiar apuntes o consultar manuales en la biblioteca. Todo ello suele resolverse a golpe de clic, ¿por qué con los diccionarios tendría que ser diferente?

Más allá de las cualidades intrínsecas de la obra lexicográfica, el éxito o alto grado de aceptación del DBO depende fundamentalmente de tres factores: accesibilidad, inmediatez y carácter gratuito (Mann 2014: 3, Nied Curcio 2015: 449). El formato online permite acceder al contenido lexicográfico de forma rápida e inmediata desde diferentes dispositivos, entre ellos, el teléfono móvil, con frecuencia, un smartphone o teléfono inteligente. Prueba de ello es la existencia de una multiplicidad de Apps de DBO para el par de lenguas alemán-español, generalmente gratuitas (cf. Meliss 2013), una circunstancia que ha contribuido muy significativamente a su popularización entre el alumnado.

Sin duda, se trata de tres propiedades valoradas muy positivamente entre los aprendices; sin embargo, ¿qué pasaría si la interfaz de usuario del DBO fuera poco intuitiva o eficaz?, ¿si tuviéramos que hacer numerosos clics para llegar al equivalente en la lengua meta y hacer frente a innumerables ventanas emergentes?, ¿si la página cargara mal, la conexión fuera lenta o tuviéramos que pagar para acceder a una versión premium? y, por lo tanto, ¿qué pasaría si se vieran comprometidos los parámetros de accesibilidad, inmediatez y carácter gratuito? Seguramente dejaríamos de utilizar ese DBO, simplificaríamos la búsqueda o, directamente, dejaríamos la consulta para otro momento.

Este patrón de comportamiento nos hace reflexionar sobre la incidencia real de estos tres factores en el proceso de selección de la obra lexicográfica y es que, desde nuestro punto de vista, no solo condicionan o favorecen la selección del DBO frente a un diccionario en formato papel, sino que, además, determinan la selección de un 
DBO en concreto sin atender a otros factores relacionados con la calidad o grado de adecuación de la obra lexicográfica a las necesidades comunicativas de aquellos que la consultan.

\section{APRENDICES DAF Y DBO, ¿POR QUÉ DEBERÍAN UTILIZARLOS?}

Junto a los factores de accesibilidad, inmediatez y carácter gratuito destacan otros que, a priori, no suelen influir en el usuario a la hora de seleccionar una herramienta lexicográfica en formato online, pero que, sin duda, suponen un valor añadido, al menos desde el punto de vista del lexicógrafo. Se trata, entre otros, de la simplificación del proceso de elaboración del diccionario o la agilización de su distribución (Mann 2014: 3).

En la actualidad existen un gran número de herramientas de software que ofrecen soluciones estandarizadas para la creación y edición de diccionarios online. Por otro lado, en lo que concierne a la distribución, hay que tener en cuenta que la naturaleza hipertextual de los diccionarios online facilita y agiliza su acceso y distribución en un mercado global, cada vez más receptivo al consumo de productos virtuales, lo cual no es una excepción en el ámbito académico o educativo. Además, el formato online ofrece nuevas posibilidades técnicas que permiten superar algunas de las limitaciones que presenta el formato en papel, como, por ejemplo, agilizar el proceso y la frecuencia de actualización, su carácter multimedial, la conexión a otros recursos lexicográficos e, incluso, la posibilidad de convertirse en un espacio colaborativo.

A continuación exponemos de forma sistemática algunas de estas propiedades, que, desde nuestro punto de vista, tendrían que ser tenidas en cuenta por los usuarios a la hora de seleccionar un diccionario online y, en concreto un DBO para el par de lenguas alemán-español.

\subsection{Actualización inmediata}

El formato online otorga a los diccionarios un carácter dinámico, lo que tiene una consecuencia directa en su proceso de actualización. 
La información lexicográfica puede ser actualizada en cualquier momento sin necesidad de someterse a los tiempos de la planificación editorial (Harvey 2000: 85). Tal y como afirma de Schryver (2003: 157), se trata de una de las propiedades más significativas del diccionario online en cuanto que lo convierte en "the ultimate dynamic repository of knowledge."

Por otra parte, en el caso de los errores, ya sean faltas de ortografía, erratas o errores de traducción, el formato online agiliza también su corrección, lo que evita que puedan dificultar el proceso de decodificación de la información lexicográfica por parte del usuario. De hecho, en el caso de Pons-online y LEO, dos de los DBO más populares entre los aprendices hispanohablantes de DaF en el contexto universitario español ${ }^{4}$ (Meliss 2015: 409), se anima a los propios usuarios a poner en conocimiento del equipo de redacción cualquier error que identifiquen en el diccionario. Esta posibilidad nos permite introducir una de las características más significativas de los DBO frente a los diccionarios en formato papel: el carácter colaborativo.

\subsection{Carácter colaborativo}

Aunque la participación activa de los usuarios en el proceso de elaboración del diccionario se remonta al siglo XIX (cf. Thier 2014), y, por lo tanto, al formato tradicional, la localización de los diccionarios en el espacio virtual ha multiplicado las opciones de colaboración. Tomando como referencia Pons-online y LEO como ejemplos representativos de DBO para el par de lenguas alemán-español, se identifican varias posibilidades. Por un lado, como apuntamos anteriormente, la notificación de errores. Ambos diccionarios ponen a disposición del usuario un formulario, para cuyo uso, en el caso de Pons-online, es necesario registrarse. LEO, por el contrario, facilita la interacción usuario-diccionario eludiendo esta fase previa. LEO intensifica además su potencial colaborativo, ya que anima a los usuarios a dejar su dirección de correo electrónico en el formulario en el caso de estar interesados en una respuesta por parte del equipo de redacción. Por otra parte, en esta misma línea, LEO ofrece la posibilidad de escribir un correo si se identifica un error general en la página web o en la $A p p$ que dificulte la accesibilidad al recurso. Pons- 
online también ofrece este servicio bajo la etiqueta Feedback, a través de la cual el usuario puede realizar las siguientes acciones: "quiero alabar esta página," "he encontrado un error," "quiero hacer una propuesta" y "deseo formular una crítica."

Otra forma de colaboración que se identifica en LEO es la participación en un foro sobre cuestiones lingüísticas y culturales entre los usuarios del diccionario. Pons-online no ofrece este servicio; sin embargo, permite al usuario proponer una nueva entrada, que tras ser revisada por el equipo de redacción podrá incluirse en el diccionario (en este caso se hace referencia a que se trata de una entrada elaborada por un usuario). LEO, por el contrario, no permite la creación de entradas, sino la cesión de listas de vocabulario en forma de donación. Estas nuevas formas de colaboración acercan el diccionario a otros espacios colaborativos web de uso frecuente y, en esta medida, favorece la identificación del usuario con el producto lexicográfico (Abel y Meyer 2016: 251).

\subsection{Multimedialidad}

Una de las grandes ventajas que reporta la naturaleza hipertextual de los DBO es la posibilidad de acceder a material auditivo y audiovisual desde el propio artículo lexicográfico, como por ejemplo a un archivo de audio. Esta herramienta permite al usuario resolver dudas concretas sobre la pronunciación de una palabra o grupo fónico, así como practicar la pronunciación de forma autónoma mediante la repetición e imitación a partir de un input lingüístico.

En el contexto DaF, esta información es especialmente valiosa, ya que puede ayudar al aprendiz a desambiguar dos acepciones y, así, facilitar el proceso de selección del equivalente. Pensemos por ejemplo en los verbos prefijados del alemán del tipo durchfahren. Estos verbos presentan una variante separable, en la que el acento recae sobre el verbo (durch fahren = pasar, conducir sin parar), y una variante inseparable ('durchfahren = recorrer, atravesar), en la que el acento recae sobre el prefijo. A través del input auditivo y con unos conocimientos gramaticales básicos, el aprendiz puede discernir entre una y otra acepción y asimilar, a su vez, su correcta pronunciación, algo que le facilitará su codificación y decodificación en contextos comunicativos orales. 


\subsection{Acceso a información lexicográfica adicional}

En muchas ocasiones, los DBO proponen enlaces de acceso a recursos lexicográficos externos. Esta particularidad no solo permite profundizar en la búsqueda, sino también personalizarla. Se trata de lo que Mann (2014: 3) denomina Benutzeradaptivität en relación a la capacidad del diccionario online para adaptarse a las necesidades comunicativas del usuario. Imaginemos que un aprendiz de alemán de nivel avanzado, una vez decodificado el verbo durchfahren y seleccionado el equivalente en la lengua materna, está interesado por su combinatoria con el fin de producir un texto escrito: "¿con qué palabras aparece?, ¿cuáles son las más frecuentes?” Aparte de los ejemplos propuestos por el artículo lexicográfico, la información que ofrecen los diccionarios bilingües a este respecto suele ser bastante limitada. El usuario tiene que buscar nuevos recursos para continuar con su consulta, a los cuales puede acceder directamente desde el diccionario online, como por ejemplo sucede en Pons-online o LEO.

La posibilidad de personalizar la búsqueda implica una toma de conciencia por parte del usuario sobre sus necesidades comunicativas más allá de la búsqueda de un equivalente: "ya sé lo que significa el verbo durchfahren, pero ¿puedo hacer algo con él?, ¿'soy capaz de utilizarlo?, ¿`é conjugarlo?, ¿̇en qué contexto se utiliza?” Este proceso reflexivo favorece el desarrollo de la propia conciencia lingüística, la autonomía en el proceso de aprendizaje de la lengua extranjera, así como la concepción de la obra lexicográfica como una herramienta de aprendizaje y no solo de consulta.

\subsection{Lectura no lineal y exportación de la información}

Con ello nos referimos a la organización no secuencial de la información y a la posibilidad de saltar de una parte a otra del texto sin necesidad de seguir un orden predeterminado según los intereses del usuario. Este decide si quiere acceder a recursos externos, si quiere escuchar el archivo de audio o si, por ejemplo, quiere acudir a la entrada lexicográfica del equivalente en la lengua meta. La nolinealidad de los DBO permite al usuario tomar decisiones sobre el proceso de consulta, promoviendo con ello un uso más consciente del diccionario. 
Por otra parte, cabe también destacar la posibilidad de exportar la información lexicográfica. Podemos copiarla y pegarla y, una vez hecho eso, por ejemplo, en un documento Word, podemos recortarla, subrayarla, cambiarle el tamaño, el color, etc. En ocasiones, como por ejemplo en Pons-online y LEO, se ofrece también la posibilidad de crear una lista de palabras con el fin de elaborar un entrenador de vocabulario personalizado. Esta particularidad pone especialmente de relieve el valor del diccionario en formato online como herramienta de aprendizaje y su capacidad para adaptarse a las necesidades concretas del usuario.

\subsection{Simplificación del proceso de consulta}

El formato online ofrece una serie de facilidades a la hora de llevar a cabo el proceso de consulta. En primer lugar, no se supedita al orden alfabético, ya que permite escribir la palabra directamente en el buscador. Otra de las ventajas es que, por lo general, hay cierta tolerancia a los errores ortográficos y tipográficos, de manera que el usuario puede llegar a la entrada lexicográfica sin haber escrito correcta o completamente la palabra que quiere consultar. En el caso del alemán esta opción es muy interesante debido a sus particularidades ortográficas, como por ejemplo el uso de las mayúsculas en los sustantivos o el uso de la diéresis en vocales en las que el español no la admite (ä y ö), lo que, en un nivel inicial, puede dificultar el proceso de consulta lexicográfica a los aprendices hispanohablanes de DaF.

Otra opción interesante es la de introducir en el buscador combinaciones de palabras como colocaciones del tipo dar un paseo (einen Spaziergang machen), cuya codificación en la lengua meta suele ser especialmente problemática para los aprendices. Si realizamos esta consulta en un diccionario en formato papel tendríamos que buscar el sustantivo y, a continuación, leer atentamente todo el artículo lexicográfico con el fin de encontrar el verbo con el que conforma la unidad plurilexemática. En este caso, además, tanto Pons-online como LEO permiten alterar el orden de las palabras, de manera que el usuario obtendría el mismo resultado si introdujera en el buscador la secuencia <machen einen Spaziergang>. 


\section{HÁBITO VERSUS COMPETENCIA O DE CÓMO UTILIZAN LOS APRENDICES DAF LOS DBO}

A pesar del alto grado de aceptación del DBO, es frecuente la identificación de carencias de uso entre los usuarios, entre ellos los estudiantes universitarios. El factor condicionante, al igual que sucede con los diccionarios en papel, es, fundamentalmente, el desconocimiento de "esos elementales principios relativos a su contenido y a su estructura" (Porto Dapena 2002: 10).

En el caso concreto del proceso de enseñanza-aprendizaje de DaF, a pesar de la gran oferta y paulatina mejora de los recursos lexicográficos (Fuentes 2015: 295), este escenario no dista mucho de la realidad vigente en las aulas universitarias. Desde nuestra propia experiencia docente, el origen de gran parte de los errores de uso del diccionario y, en el caso que aquí nos ocupa, del DBO parte de la arbitrariedad a la hora de seleccionar la obra lexicográfica.

Con frecuencia, los estudiantes, más que una recomendación, esperan una directriz clara y concisa por parte del docente, materializada en forma de enlace directo. Por lo general, independientemente de que hayan recibido una orientación o formación más o menos específica, los estudiantes suelen dedicar poco tiempo a reflexionar sobre el grado de adaptabilidad y adecuación de la obra lexicográfica a sus necesidades concretas.

Esta falta de interés por el proceso de selección puede explicarse aludiendo a diversos factores. En primer lugar, el desconocimiento por parte de los usuarios de las posibilidades de explotación del recurso acorde con su valor como obra de consulta y aprendizaje, así como del aprovechamiento de la información implícita que pone a su disposición. En segundo lugar, derivado del anterior, la distancia entre el usuario como consumidor y el diccionario como objeto de consumo. Si el usuario, más allá de su carácter accesible e inmediato, conociera las características del DBO, entre ellas las posibilidades de interacción y colaboración, seguramente la identificación de este con el producto lexicográfico sería mayor. Por último, aunque a priori suponga una gran ventaja, el carácter gratuito de la mayoría de los DBO puede hacer que el usuario, en cierta manera y quizás de forma inconsciente, 
infravalore la calidad de la información lexicográfica del diccionario al desaparecer la segunda variable del binomio calidad-precio.

En cuanto al uso en concreto que hacen los aprendices de DaF de los DBO, derivado o no de una selección no adecuada de la obra lexicográfica y de acuerdo a los resultados obtenidos en la encuesta DICONALE-DIC, entre los errores de uso más frecuentes destaca la elección equivocada del lema o equivalente (Fernández Méndez et al. 2016: 84), con frecuencia como resultado de un proceso de consulta precipitado. Otros errores frecuentes de uso del DBO son la elección errónea de la estructura argumental que acompaña al lema y el uso incorrecto de la estructura sintáctica (84). Estos errores de uso pueden incidir en la actuación lingüística del usuario (performance) y provocar faltas, no solo en el ámbito de la producción o recepción en la lengua extranjera, sino también en el de la interacción y mediación, que, de no ser identificadas, pueden derivar en errores de uso de la lengua, por lo general de carácter léxico y morfosintáctico, pero también de carácter discursivo o de pronunciación.

A continuación, proponemos tres ejemplos que ponen de manifiesto la incidencia de los errores de uso del diccionario, en este caso DBO, en la performance de los aprendices. Se trata de ejemplos reales contextualizados en una actividad de producción escrita llevada a cabo en la asignatura Alemán V (nivel A2.2) del Grado en Turismo de la Universidad de Salamanca durante el curso académico 2016-2017:

(1) *Das Hoch:eitsmenü besteht aus drei Tellern und Nachtisch.

(2) *Das Brautpaar hat einen Flug nach New York reserviert.

(3) *Den Nachtisch hat den Gästen nicht gefallen.

En el ejemplo (1), en español el menú de boda se compone de tres platos y un postre, el aprendiz no selecciona el equivalente adecuado de la palabra plato. En español, plato hace referencia al recipiente (plato hondo, plato llano, etc), al alimento que se sirve en el recipiente (plato soso, plato sabroso, etc.) y, como en el ejemplo, a cada una de las partes que componen un menú. El alemán, por el contrario, diferencia entre Teller para el recipiente, Gericht para los alimentos y Gang para los "platos" del menú. En este caso, por lo tanto, el equivalente adecuado sería Gang y no Teller (Das Hochzeitsmenü besteht aus drei Gängen und 
Nachtisch.). En el ejemplo (2), en español los novios han reservado un vuelo para Nueva York, atendiendo a la estructura argumental del verbo y, en concreto, a la realización semántica de los argumentos, el equivalente adecuado del verbo reservar sería buchen y no reservieren, ya que en alemán el verbo reservieren selecciona sustantivos como mesa (es. reservar una mesa en un restaurante; de. einen Tisch im Restaurant reservieren) o asiento (es. reservar un asiento en el tren; de. im Zug einen Sitzplatz reservieren), mientras que el verbo buchen coloca con sustantivos como vuelo o viaje (de. einen Flugleine Reise buchen). Por último, en el ejemplo (3), en español a los invitados no les ha gustado el postre, el usuario hace un uso incorrecto de la estructura sintáctica del verbo gefallen, ya que, en alemán, al igual que en español (gustar), se trata de un verbo intransitivo, por lo que no es correcto el uso del acusativo en den Nachtisch, siendo la forma correcta el uso del nominativo, der Nachtisch. Estas tres faltas en el ámbito de la producción escrita podrían haberse evitado si el aprendiz hubiera hecho un uso correcto de la obra lexicográfica, a la que, cabe destacar, tenía acceso durante el desarrollo de la tarea, ya sea con una lectura más exhaustiva del artículo lexicográfico, incluyendo ejemplos, o mediante la correcta interpretación de las marcas lexicográficas.

En otra actividad, se les pidió a los estudiantes que, por grupos, describieran brevemente las siguientes festividades: Oktoberfest, Schützenfest y Fasching, partiendo para ello de la información que ponía a su disposición el diccionario, en este caso en concreto Pons-online. En un principio cada grupo se limitó a ofrecer el equivalente en la lengua materna hasta que se les indicó de forma explícita la posibilidad de acceder a información adicional desde el propio artículo lexicográfico, algo que desconocían hasta el momento a pesar de haber usado esta misma herramienta lexicográfica con anterioridad.

Teniendo en cuenta los ejemplos anteriores, los errores de uso que suele cometer un usuario de un DBO, aquí aprendices de DaF, no distan mucho de los que comete cuando consulta un diccionario en formato papel $^{5}$ (Nied Curcio 2015: 450). Este hecho nos hace reflexionar sobre la incidencia real de la lexicografía electrónica en los hábitos de consulta de los usuarios para llegar a la conclusión de que, en la mayoría de los casos, su desarrollo, tanto a nivel teórico como práctico en forma de nuevos recursos cada vez más intuitivos y de 
mayor calidad informativa, no se sucede en consonancia con una pedagogía lexicográfica en las aulas y, por lo tanto, con la adquisición y desarrollo por parte de los usuarios de unas competencias lexicográficas específicas (Egido Vicente y Meliss 2017: 48), que, tal y como afirman Kozyrev y Chernjak (2007: 157), son componente fundamental de la competencia lingüística.

Con frecuencia, los estudiantes, reproduciendo una serie de hábitos adquiridos en etapas posteriores, demuestran escasos conocimientos sobre el manejo y posibilidades de aprovechamiento de los DBO, de algo que, además, no suelen ser conscientes. Desde nuestro punto de vista, este es precisamente uno de los factores que inciden negativamente en el desarrollo de la competencia lexicográfica en el contexto de la enseñanza superior. Por lo general, tanto por parte de los aprendices como de los docentes, el uso correcto del diccionario, independientemente de su formato y tipología, suele considerarse una tarea aprendida en esta fase del sistema educativo, sobre todo teniendo en cuenta que los currículos de Educación Primaria y Educación Secundaria contemplan la didáctica lexicográfica, ya sea para la lengua materna como para la extranjera (Egido Vicente 2018: 136).

\section{CONCLUSIONES}

La incorporación de las nuevas tecnologías y el desarrollo de una lexicografía centrada en las necesidades del usuario ha propiciado la creación de una nueva generación de diccionarios cada vez más intuitivos y manejables. Su contextualización en el marco de una sociedad digitalizada ha traído consigo una democratización de los recursos lexicográficos, materializada en la existencia de una amplia oferta. El usuario, en este caso el estudiante universitario, acostumbrado al uso de plataformas virtuales en su vida cotidiana, prefiere, por lo general, los diccionarios en formato online frente a los diccionarios tradicionales en formato papel. En el contexto DaF, una vez superada la manida discusión teórica en torno al marcado carácter decodificador de la obra lexicográfica bilingüe, los aprendices, especialmente en los niveles inicial e intermedio, optan por la utilización de un DBO. 
Partiendo de esta premisa y de su entorno cultural y educativo, los docentes e incluso los propios usuarios dan por supuesto el adecuado manejo de la herramienta lexicográfica, algo que, como hemos podido comprobar, no siempre se corresponde con la realidad. Las carencias de los usuarios en el ámbito de las competencias lexicográficas se ponen claramente de manifiesto con la selección precipitada del recurso, en la que, con frecuencia, priman factores como la accesibilidad, inmediatez y carácter gratuito frente a otros relacionados con la calidad de la información lexicográfica y su grado de adecuación.

En cuanto al proceso de consulta, se observa como los usuarios, a pesar de las posibilidades que ofrece el formato hipertextual, siguen cometiendo los mismos errores que con los diccionarios en formato papel, derivados generalmente de una lectura más bien somera del artículo lexicográfico y de un desconocimiento o errónea interpretación de las marcas lexicográficas. También destaca un escaso aprovechamiento del recurso en lo referente al acceso a la información lexicográfica adicional, así como a su carácter multimedial y colaborativo. Tomando en consideración este patrón de comportamiento, se llega a la conclusión que los avances llevados a cabo en el campo de la lexicografía digital, así como en el de la Wörterbuchbenutzungsforschung, especialmente en el proceso de enseñanza-aprendizaje de DaF en el contexto de la enseñanza superior, no se han materializado en la formación de usuarios competentes, no al menos en la medida esperable. Para suplir esta carencia es necesario que los usuarios tomen conciencia de su desconocimiento y asuman la necesidad de emprender un proceso de reflexión en torno a los hábitos adquiridos. Este proceso debe ser propiciado en el aula a partir de la incorporación de una didáctica lexicográfica actual que contemple las particularidades del formato online y que ponga su atención en las necesidades concretas de los usuarios de forma individualizada.

Además de la descripción de las características y propiedades de los recursos lexicográficos, y con el fin de sacarle el mayor partido posible, es fundamental hacer hincapié en la importancia del proceso de selección del diccionario. Para ello, es necesario promover el espíritu crítico de los aprendices frente a la obra lexicográfica, así 
como su valor como obra de consulta y aprendizaje. Desde nuestro punto de vista, la puesta en marcha de esta toma de conciencia por parte del usuario y el consecuente desarrollo de las competencias lexicográficas parte del convencimiento del docente como resultado de una profunda reflexión sobre sus hábitos lexicográficos, primero como usuario y, posteriormente, en el contexto de la enseñanzaaprendizaje de la lengua extranjera, lo cual solo será posible a través de una formación específica.

Bajo esta premisa, consideramos necesario que la didáctica lexicográfica forme parte del proceso de formación del profesorado de lenguas, tanto maternas como extranjeras, en los diferentes niveles de enseñanza y como paso previo y fundamental a la formación de futuros usuarios competentes de la obra lexicográfica y, en concreto, del DBO.

\section{NOTAS}

${ }^{1}$ El presente artículo se desarrolla en el marco del proyecto de investigación COMBIDIGILEX (La combinatoria en paradigmas léxico-semánticos en contraste. Estudio empírico y digitalización para el aprendizaje de lenguas extranjeras en contextos germanoiberorrománicos) financiado por el Ministerio de Economía y Competitividad (FFI2015-64476-P) y desarrollado en la Universidad de Santiago de Compostela.

${ }^{2}$ Con este término nos referimos a la disciplina más joven de la lexicografía impulsada en los años 90 por el germanista Herbert Ernst Wiegand en torno al uso del diccionario (Wiegand 1998, 2008 y 2010). En este caso en concreto, nos referimos a la encuesta UDALPE (Domínguez Vázquez et al. 2013) y DICONALE-DIC (Meliss 2015) sobre el estudio de los hábitos lexicográficos de aprendices hispanohablantes de DaF. La encuesta UDALPE se lanza exclusivamente en el ámbito universitario (Domínguez Vázquez et al. 2013: 141). En ella participan estudiantes de Filología Alemana y de Traducción con alemán como primera lengua extranjera. La encuesta DICONALE-DIC, por el contrario, se lanza también a Escuelas Oficiales de Idiomas. Sin embargo, la mayoría de los encuestados provienen igualmente del ámbito universitario (Meliss 2015: 405).

${ }^{3}$ Consúltese en esta relación, entre otros, la Propuesta de 
Recomendación del Consejo relativa a las competencias clave para el aprendizaje permanente de 2018.

${ }^{4}$ Para una caracterización de Pons-online y LEO consúltense, entre otros, los artículos de Storrer (2010) y Meliss (2013).

${ }^{5} \mathrm{El}$ último ejemplo es un caso claro de desaprovechamiento o desconocimiento de la información implícita en los DBO.

\section{OBRAS CITADAS}

Abel, A. y C. M. Meyer 2016. “Nutzerbeteiligung,” en A. Klosa y C. Müller-Spitzer Carolin, eds. Internetlexikografie. Ein Kompendium. Berlin y Nueva York: de Gruyter. 249-290.

Albrecht, J. 2005. Übersetzung und Linguistik. Tübingen: Narr. de Schryver, G.-M. 2003. "Lexicographers' dreams in the electronicdictionary age". International Journal of Lexicography, 16, 2: 143-199.

Domínguez Vázquez, M. J., M. Mirazo Balsa y V. Vidal Pérez 2013. "Wörterbuchbenutzung: Erwartungen und Bedürfnisse. Ergebnisse einer Umfrage bei Deutsch lernenden Hispanophonen," en M. J. Domínguez Vázquez, ed. Trends in der deutsch-spanischen Lexikographie. Frankfurt am Main: Peter Lang. 135-172.

Egido Vicente, M. 2018. “La competencia lexicográfica en el proceso de enseñanza-aprendizaje de lenguas extranjeras en el contexto de enseñanza superior. ¿Una asignatura pendiente?” en M. Ellison, M. Pazos Anido, P. Nicolás Martínez y S. Valente Rodrigues, eds. As linguas estrangeiras no ensino superior: propostas didácticas e casos em estudo. Porto: FLUP. 125-139.

Egido Vicente, M. y M. Meliss 2017. “¿Cómo usar diccionarios bilingües en línea? Ejemplos para el desarrollo de las competencias lexicográficas en la enseñanza del alemán como lengua extranjera". RILE 7: 31-53. Consultado el 15/03/2018 https://revistes.urv.cat/index.php/rile/article/view/1911.

Engelberg, S. y L. Lemnitzer 2009. Lexikographie und Wörterbuchbenutzung. Tübingen: Staufenburg.

Fernández Méndez, M., M. Franco Barrios e I. Sueiro Orallo 2016. “El uso de los diccionarios de/para ELE y DaF. Análisis de una encuesta desde una perspectiva contrastiva," en F. Robles i 
Sabater, D. Reimann y R. Sánchez Prieto, eds. Sprachdidaktik Spanisch - Deutsch Forschungen an der Schnittstelle von Linguistik und Fremdsprachendidaktik. Tübingen: Narr. 73-92.

Fuentes Morán, M. T. 2015. "Representación del anisomorfismo en diccionarios bilingües alemán-español," en M. Meliss y B. Pöll, eds. Aktuelle Perspektiven der kontrastiven Sprachwissenschaft: Deutsch-Spanisch-Portugiesisch. Zwischen Tradition und Innovation. Tübingen. Narr. 285-297.

Gove, P. B., ed. 1963. Webster's Seventh New Collegiate Dictionary. Springfield: G. \& C. Merriam Company.

Harley, A. 2000. "Cambridge Dictionaries Online," en U. Heid, S. Evert, E. Lehmann y C. Rohrer, eds. Proceedings of the Ninth Euralex Interational Congress EURALEX 2000, Stuttgart 8-12 August 2000. 85- 88. Consultado el 15/03/2018 http://www.euralex.org/ elx_proceedings/Euralex2000.

Höfler, E. 2017. "Mit YouTube-Stars Fremdsprachen lernen. Eine interdisziplinäre Annäherung," en A. Cortí y J. Wolf eds. Romanistische Fachdidaktik. Salzburger Beiträge zur Lehrer/innen/ bildung: Der Dialog der Fachdidaktiken mit Fach-und Bildungswissenschaften. Münster/New York: Waxmann. 147-159.

Kozyrev, V. A. y V. D. Chernjak 2007. Obrazovatel'naja sreda. Jazykovaja situatsija. Rechevaja kul'tura: Monografija (Education Environment. Language Situation. Speech Culture: Monograph). St. Petersburg: Herzen University Press.

LEO: https://www.leo.org/esde/index_de.html. Consultado el $15 / 03 / 2018$.

Mann, M. 2014. "Digitale Lexikographie. Einführung in Thema und Beiträge dieses Bandes," en M. Mann, ed. Digitale Lexikographie. Ein- und mehrsprachige elektronische Wörterbücher mit Deutsch: aktuelle Entwicklungen und Analysen. Hildesheim, Zürich y Nueva York: Georg Olms Verlag. 1-12.

Martín Torres, G. 2010. El español en Alemania. El alemán en España. Subdirección General de Documentación y Publicaciones. Ministerio de Edicación.

Meliss, M. 2013. "Online-Lexikographie im DaF-Bereich: Eine erste kritische Annäherung: Bestandsaufnahme - Nutzen Perspektiven”. REAL - Revista de Estudos Alemães 4: 176- 199.

Meliss, M. 2015. "Was suchen und finden Lerner des Deutschen als Fremdsprache in aktuellen Wörterbüchern? Auswertung einer 
Umfrage und Anforderungen an eine aktuelle DaFLernerlexikographie". Info DaF 4: 401- 432.

Nesi, H. 2008. "Dictionaries in electronic form," en A. P. Cowie, ed. The Oxford History of English Lexicography. Oxford: Oxford University Press. 458- 478.

Nied Curcio, M. 2015. "Wörterbuchbenutzung und Wortschatzerwerb." Info DaF 5: 445- 468.

Pons-online: http://de.pons.com/. Consultado el 15/03/2018.

Porto Dapena, J. A. 2002. Manual de técnica lexicográfica. Madrid: Arco/Libros.

Procter, P., ed. 1978. Longman Dictionary of Contemporary English. Harlow: Longman Group.

Recomendación del Parlamento Europeo y del Consejo de 18 de diciembre de 2006 sobre las competencias clave para el aprendizaje permanente, 2006/962/CE. Consultado el 15/03/2018 https://eur-lex.europa.eu/legal-content/ES/TXT/?uri=celex \% $3^{\text {a }}$ $32006 \mathrm{H} 0962$.

Storrer, A. 2010. "Deutsche Internet-Wörterbücher: Ein Überblick," en R. H. Gouws, U. Heid, T. Herbst, S. J. Schierholz y W. Schweickard, eds. Lexicographica 26: 155-164. Berlin y Nueva York: de Gruyter.

Thier, K. 2014. "Das Oxford English Dictionary und seine Nutzer" en A. Abel y A. Klosa eds. Ihr Beitrag bitte! - Der Nutzerbeitrag im Wörterbuchprozess. 3. Arbeitsbericht des wissenschaftlichen Netwwerks Internetlexikografie (OPAL 2/2014). 62-69. Consultado el 15/03/2018 http://pub.ids-mannheim.de/laufend/ opal/pdf/ opal2014-4.pdf.

Wiegand, H. E. 1998. Wörterbuchforschung; Untersuchungen zur Wörterbuchbenutzung, zur Theorie, Geschichte, Kritik und Automatisierung der Lexikographie. Berlin: de Gruyter.

Wiegand, H. E. 2008. "Zur Wörterbuchbenutzung bei der Übersetzung. Möglichkeiten ihrer Erforschung”. Germanistische Linguistik 195-196: 1- 43.

Wiegand, H. E. 2010. "Zur Methodologie der Systematischen Wörterbuchforschung: Ausgewählte Untersuchungs- und Darstellungsmethoden für die Wörterbuchform". Lexikographica 26: 249-330.

Wilks, Y. A., B. M. Slator y L. Guthrie 1996. Electric Words: Dictionaries, Computers and Meanings. Cambridge: The MIT Press. 